\title{
Automatic Low-Cost Tool for Head 3D Modelling and Cranial Deformation Analysis in Infants
}

\author{
Inés BARBERO-GARCÍA ${ }^{1}$, José Luis LERMA ${ }^{1}$, Pablo MIRANDA ${ }^{2}$ \\ ${ }^{1}$ Photogrammetry and Laser Scanner Research Group, Universitat Politècnica de València, Spain; \\ ${ }^{2}$ Hospital Universitari i Politècnic La Fe, Valencia, Spain
}

https://doi.org/10.15221/19.009

\begin{abstract}
Cranial deformation is a problem that affects an important number of infants. It requires different treatments, varying from repositioning techniques to surgery depending on the leading cause. The evaluation of the deformation is usually carried out using simple but low-accuracy methodologies such as visual assessment and clinical measurements. Accurate methodologies such as radiological tests and combinations of cameras and scanner are available but they are invasive and/or costly.

A patent-pending smartphone-based photogrammetric tool for the analysis of cranial deformation is presented. The methodology allows the generation of head three-dimensional (3D) models of infants automatically. It is non-invasive, low-cost and can be operated by users without any photogrammetric knowledge.

The presented tool consists of a smartphone app, a coded cap that is fitted to the head of the infant and processing software that yields the 3D models.

The data acquisition can be easily carried out during the routine clinical consultation. The patient is held by an adult while the application is used to obtain the data around the infant's head. The process is similar to a video recording, and the app guides the user during the process. The tool can tolerate the patient's movement so no immobilization or sedation is required. The whole data acquisition process can be completed in a couple of minutes. After the acquisition stage, the data is processed to obtain a 3D model of the patient's head.

The accuracy and repeatability of the process have been tested under ideal conditions. The obtained accuracy was found to be better than $1 \mathrm{~mm}$. The methodology has also been tested in real conditions obtaining models useful for the evaluation of different types of cranial deformation.

The tool can be easily implemented in clinical routine. It requires minimum resources and provides a significant improvement of the techniques currently used for cranial deformation assessment.
\end{abstract}

Keywords: Anthropometry, Craniosynostosis, Photogrammetry, Plagiocephaly, Smartphone.

\section{Introduction}

Cranial deformation is a major concern as it affects, according to different studies between $20 \%$ and $47 \%$ of infants [1-3]. The patients require different treatments including repositioning, physiotherapy, the use of cranial orthosis and, in some cases, surgery.

The correct diagnosis and quantification of the deformation is an important concern in paediatric consultations. Despite its importance, commonly used methodology include visual assessment and manual measurements using measuring tape and callipers [4-6]. These methodologies have low accuracy and provide very limited information [7]. Radiological images (Computed Tomography -CTand Magnetic Resonance Imaging -MRI-) are vital for the diagnosis of craniosynostosis and they are considered the gold standard for yielding 3D models [6]. However, these tests are costly and highly invasive (sedation is often required to keep infants still and the patients are subject to radiation). For these reasons, they are only used when craniosynostosis is suspected.

Photogrammetry and 3D scanners have been proved to be a highly accurate and non-invasive alternative to obtain the head shape [5,8,9]. However, the main challenge for the obtainment of $3 \mathrm{D}$ models using these tools is the infants' movement. The existing methodologies often are composed of a complex setup of several cameras and scanners and require an important investment. As a consequence, they are not available in most clinics and hospitals and their use is not integrated as part of the clinical practice.

In this study, we present a patent-pending tool that is automatic, smartphone-based, non-invasive and can be used by non-experts. The tool only requires a special coded cap fitted to the head and a smartphone to carry out the data acquisition. Therefore, it does not require an important investment and can be easily used in any clinical practice, consultation or in a hospital/centre.

*inbargar@topo.upv.es; (+34) 963877007. Ext.: 75578; http://gifle.webs.upv.es 
A smartphone application guides the user during the whole data acquisition. The data is then uploaded to a server for processing using the developed software. The creation of the 3D models requires only a few minutes in most cases (depending on the workstation or cloud computing service).

The final model, together with the deformation parameters calculated, is made available to the user through the web. The user is allowed to take extra measurements from the models. The creation of multitemporal models for patient monitoring is also possible.

The tool can be used for the evaluation of different types of cranial deformation. In cases where only the outer information is required (i.e. positional deformation), it can be used to support diagnosis and monitor the evolution of the patient. For deformations caused by bone development (craniosynostosis), the methodology can still be used for monitoring. The tool allows reducing the number of CT and MRI tests carried out for infants at different stages, it optimises the resources and improves significantly the results given by other non-invasive, low-cost techniques.

\section{Materials and methods}

\subsection{Coded cap}

The tool is based on the use of a coded cap. This cap is made of a highly elastic material that nicely fits in the head of the patient. The cap is covered by a high number of coded markers [10], that can be automatically detected. The distribution of the markers is known and they are made of a non-elastic material, allowing its size to be constant and known (Fig. 1). Three stickers are also provided to mark three points that will be used for registration (nasion and preauricular points).
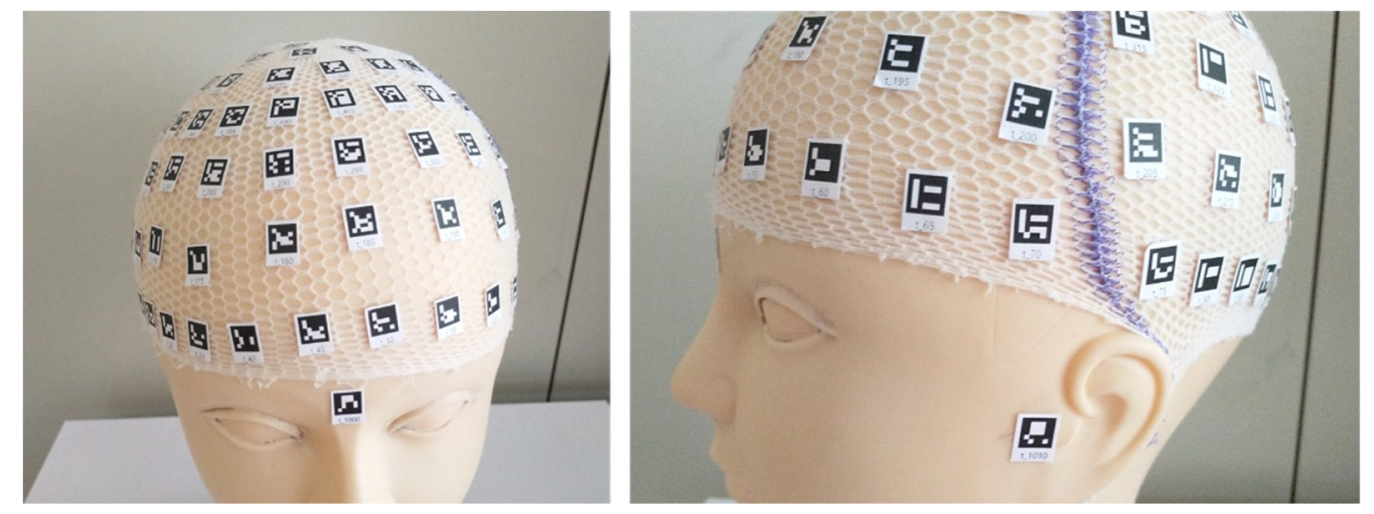

Fig. 1. Coded cap and registration points defined by stickers on a dummy.

\subsection{Smartphone application}

A smartphone application was developed for data acquisition. The app is designed so the data acquisition can be carried out by users with no photogrammetric knowledge. The interface is similar to a video recording. The whole head must be targetted by moving the smartphone around the patient. The application will detect the visible cap markers for each frame and store their position. Once the whole head is covered, the application will inform the user and send the data for processing. The only data recorded is the position of the markers for each frame.

\subsection{Data acquisition}

The data acquisition process was designed to be carried out during the usual clinical practice. It only requires a coded cap and a smartphone. No special lighting is necessary.

The patient can be held by a parent or accompanying person. This point has proven to be important to maintain children calmed and collaborative.

The fitted cap is placed and the registration points stickers are fit in theirposition. Then the smartphone app can be launched to carry out the actual data acquisition (Fig. 2). The medical staff will move the smartphone around the head. The process is similar to recording a video. Normal movement of the infant is tolerated by the tool. Even changes in the position of the infant are permitted. The time required should not exceed several minutes. This time can even be reduced with trained staff and collaborative patients. 


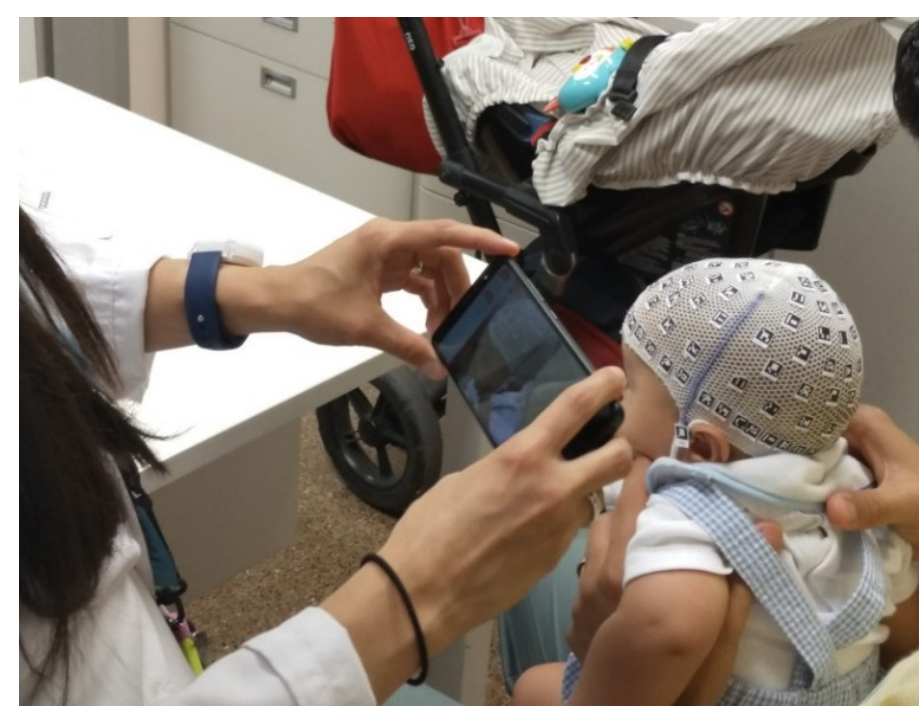

Fig. 2. Data acquisition in real conditions.

\subsection{Processing}

The creation of the 3D model can be carried out on a server or computer once the file from the smartphone app is received. The calibration and creation of the 3D model are carried out using specially developed software and the open-source software MicMac [11]. Firstly, a point cloud based on the markers is obtained. The cloud is processed to obtain a 3D model that is scaled and registered using the known marker size and the registration points. Different cranial deformation parameters are automatically obtained. The data is finally uploaded to a web page where it is visible for the user.

\subsection{Validation}

In order to validate the accuracy of the methodology, a repeatability study was carried out. Two source of errors were taken into account: photogrammetric errors and errors due to differences in the placement of the cap.

Due to the impossibility to carry out a high number of tests on a real infant, the tests were carried out in the laboratory using a dummy (Fig. 1).

A total of ten models were acquired without changing the position of the cap. Later, another ten models were created taking out the cap and stickers and putting them in the dummy again. All models were approximately registered using the registration points and later iterative closest point transformation was carried out.

The error was computed as distances between models. A sphere with its centre in the centre of the head was defined. A ray-casting algorithm was used to obtain the intersection point of the line connecting the sphere centre and each sphere point with each model. The distance between points is calculated along this line (Fig. 3).

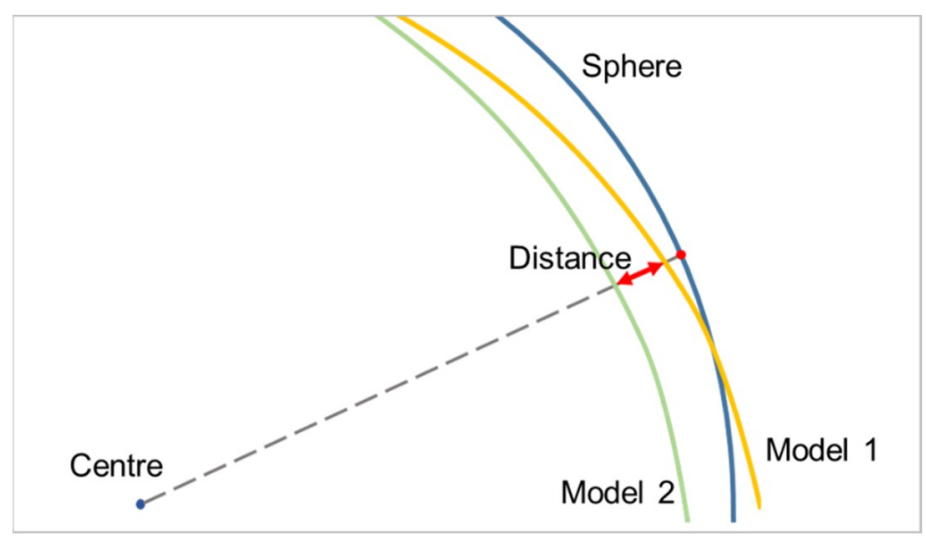

Fig. 3. Ray-casting method for calculation of distances between models. 


\section{Results}

The distances between models for both tests are shown in Table 1. In order to show the distribution of the differences, the distances are shown on a sphere (Fig. 4).

Table 1. Distances between models $(\mathrm{mm})$.

\begin{tabular}{llll} 
& Mean & Std & Maximum \\
\hline Same cap & 0.2 & 0.3 & 2.9 \\
Repositioning cap & 0.5 & 0.5 & 3.4
\end{tabular}

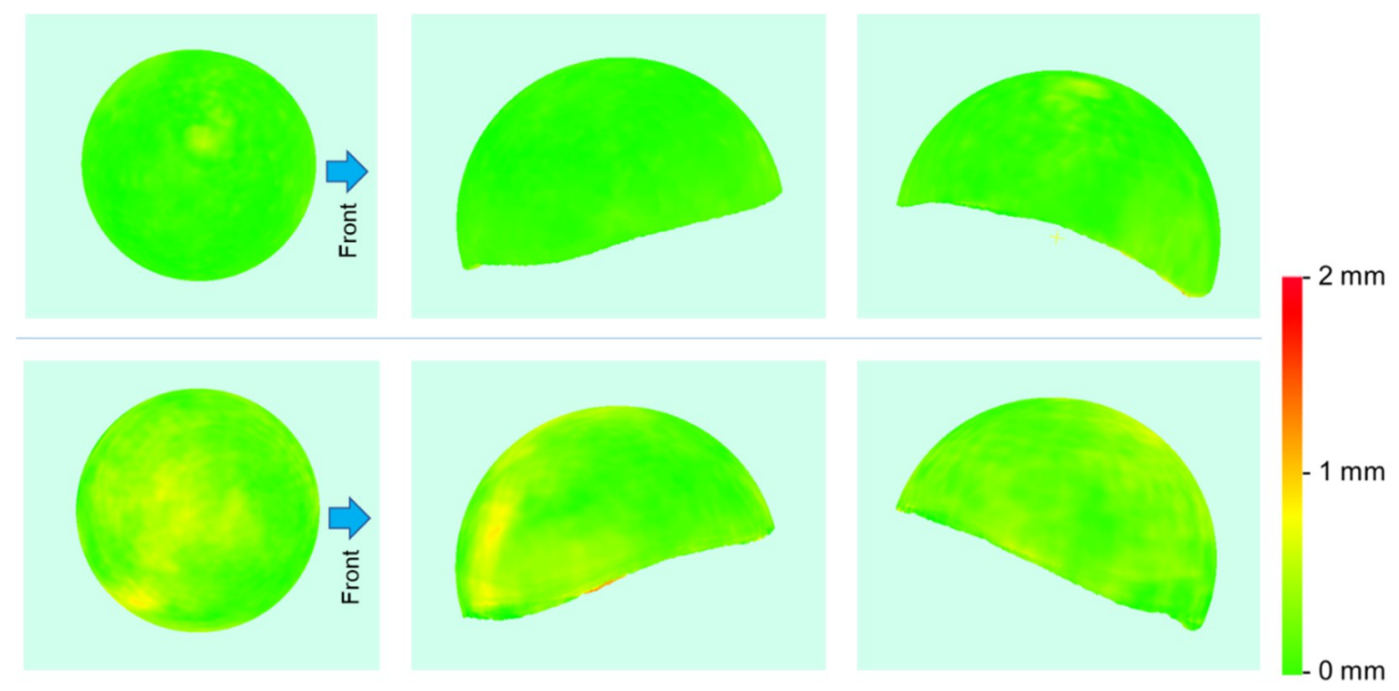

Fig. 4. Mean distance between models projected on the sphere. For the same cap (top) and repositioning the cap between data acquisitions (bottom).

Results show that differences between models are below $1 \mathrm{~mm}$ even when the cap is repositioned between acquisitions, the maximum error is $3.4 \mathrm{~mm}$. When the cap is not repositioned, most differences are below $0.5 \mathrm{~m}$ with a maximum error of $2.9 \mathrm{~mm}$. The mean values are negligible for cranial deformation analysis, however, maximum errors can be considered high. In Figure 5, the location of the maximum error areas can be examined. In both tests, the highest errors are located in small areas at the edge of the models. Other small errors can be found in the top (probably the cap is not so well fitted in that area) and in a small area for the repositioning cap test. All these errors are below $1 \mathrm{~mm}$ of difference between models and affect small areas, so their influence in the calculation of the deformation parameters will be limited.

\subsection{Real applications}

The photogrammetric tool has been tested in common clinical practice. It has been proved to be useful to identify and evaluate different types of cranial deformation (Fig. 5). It can be used for the diagnosis of plagiocephaly, and also for evaluation of the evolution of different types of already diagnosed craniosynostosis. 


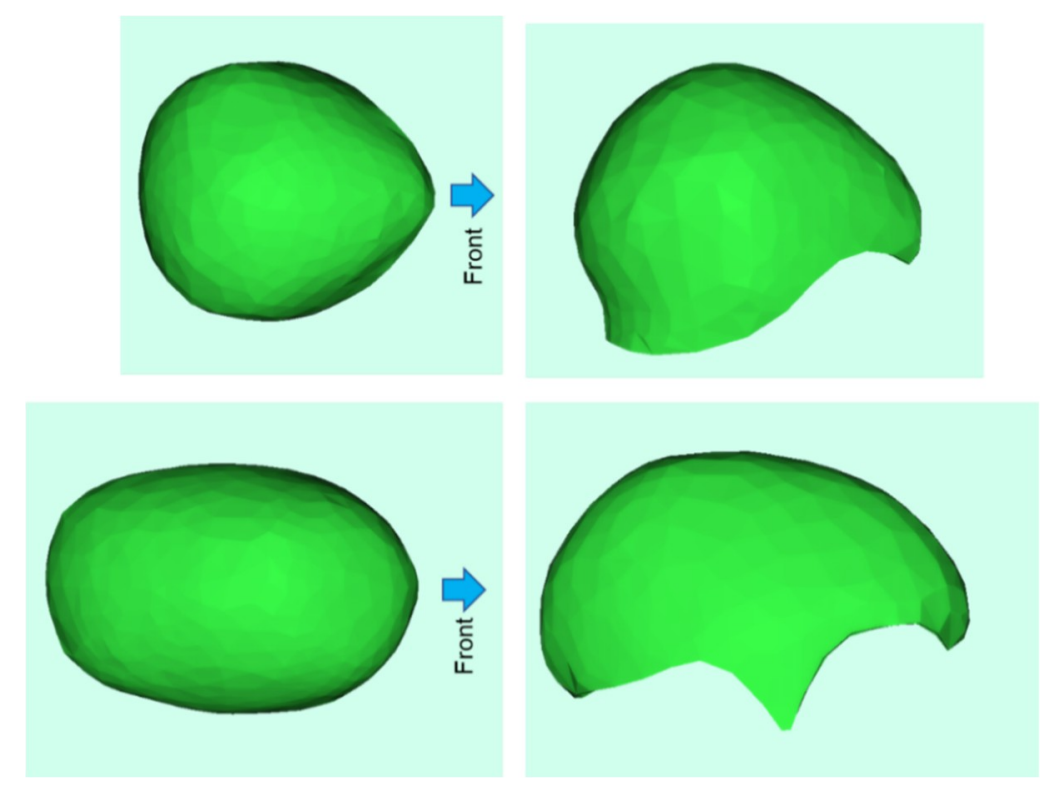

Fig. 5. Cranial 3D models obtained in real clinical conditions for a patient with trigonocephaly (top) and scaphocephaly (bottom).

\section{Discussion}

A novel low-cost methodology for measuring head shapes under real clinical practice has been presented. The repeatability error of the photogrammetric tool that yields 3D models can be considered to be below $1 \mathrm{~mm}$ even when the cap is repositioned. The photogrammetric error without changes in the cap is below $0.5 \mathrm{~mm}$. The obtained results match the required accuracy for the given application. The commonly used callipers and measurement tapes have a maximum precision of $1 \mathrm{~mm}$. Visual tolerance for cranial and face asymmetry is considered approximately $2 \mathrm{~mm}$ [12].

The obtained precision confirms that a limited number of markers is enough for an accurate representation of the head, simplifying the obtainment of 3D models. In addition, the computational cost is dramatically reduced.

The use of 3D models instead of a very limited number of measurements is an important improvement for the evaluation of cranial deformation. Models can be used to obtain commonly used measurement and indexes, and comparisons with ideal shapes [5,13].

The methodology is also capable of obtaining real models in clinical conditions. The models allow for quantification and evaluation of different types of deformation.

More research will be carried out in the future to evaluate the accuracy and repeatability of the tool in real clinical conditions. However, the data already obtain shows similar results to that obtained in ideal conditions.

The methodology presented herein can be adapted and extended for modelling and monitoring other body parts.

\section{Conclusion}

Cranial deformation is an important problem with high prevalence among infants. The most common techniques for diagnosis have low accuracy and provide very limited information. Most accurate techniques are available but they are not integrated in the common clinical practice, usually due to high costs. The lack of commonly used accurate methodologies hampers the correct evaluation of the patients and the effectivity of the different treatments.

The presented photogrammetric tool is low-cost and non-invasive and allows the obtainment of cranial 3D models of infants during the usual clinical practice. It only requires a fitted cap and a smartphone for the data acquisition and the patient can be awake and moving during the process. The app is designed to be used by medical staff with no knowledge of photogrammetry, so no technician is required for the data acquisition. In summary, a patent-pending, quick, easy-to-use smartphone-based photogrammetric tool is ready to be used for head 3D modelling, from which cranial deformation analyses can be metrically delivered. 


\section{Acknowledgements}

Funding for this project was provided by Instituto de Salud Carlos III and European Regional Development Fund (FEDER), project number PI18/00881 and by Generalitat Valenciana, grant number ACIF/2017/056.

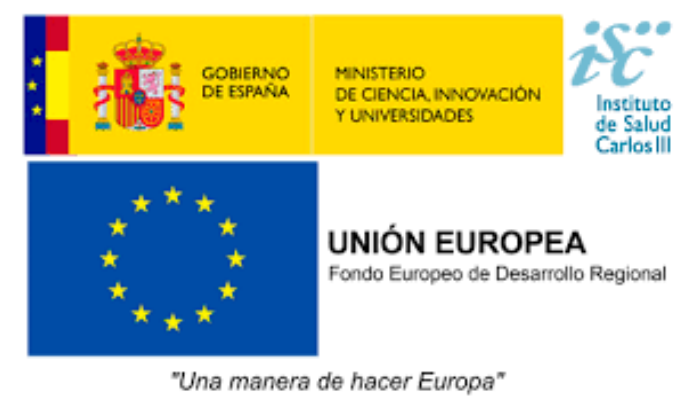

\section{References}

[1] A. Mawji, A. R. Vollman, J. Hatfield, D. A. McNeil, and R. Sauvé, "The incidence of positional plagiocephaly: a cohort study.," Pediatrics, vol. 132, no. 2, pp. 298-304, 2013, https://doi.org/10.1542/peds.2012-3438.

[2] T. S. Glasgow, F. Siddiqi, C. Hoff, and P. C. Young, "Deformational plagiocephaly : Development of an objective measure and determination of its prevalence in primary care," Journal of Craniofacial Surgery, 2007, https://doi.org/10.1097/01.scs.0000244919.69264.bf.

[3] A. E. Bialocerkowski, S. L. Vladusic, and C. Wei Ng, "Prevalence, risk factors, and natural history of positional plagiocephaly: a systematic review," Dev. Med. Child Neurol., vol. 50, no. 8, pp. 577586, 2008, https://doi.org/10.1111/j.1469-8749.2008.03029.x.

[4] C. A. A. Beaumont et al., "Three-dimensional surface scanners compared with standard anthropometric measurements for head shape," J. Cranio-Maxillofacial Surg., vol. 45, no. 6, pp. 921-927, 2017, https://doi.org/10.1016/j.jcms.2017.03.003.

[5] J. W. Meulstee et al., "A new method for three-dimensional evaluation of the cranial shape and the automatic identification of craniosynostosis using 3D stereophotogrammetry," Int. J. Oral Maxillofac. Surg., vol. 46, no. 7, pp. 819-826, 2017, https://doi.org/10.1016/j.ijom.2017.03.017.

[6] M. H. Siegenthaler, "Methods to Diagnose, Classify, and Monitor Infantile Deformational Plagiocephaly and Brachycephaly: A Narrative Review," J. Chiropr. Med., vol. 14, no. 3, pp. 191204, 2015, https://doi.org/10.1016/j.jcm.2015.05.003.

[7] S. Nahles, M. Klein, A. Yacoub, and J. Neyer, "Evaluation of positional plagiocephaly: Conventional anthropometric measurement versus laser scanning method," J. CranioMaxillofacial Surg., vol. 46, no. 1, pp. 11-21, 2018, https://doi.org/10.1016/j.jcms.2017.10.010.

[8] I. Barbero-García, J. L. Lerma, P. Miranda, and Á. Marqués-Mateu, "Smartphone-based photogrammetric 3D modelling assessment by comparison with radiological medical imaging for cranial deformation analysis," Measurement, vol. 131, pp. 372-379, 2019, https://doi.org/10.1016/j.measurement.2018.08.059.

[9] D. A. Mendonca, S. D. Naidoo, G. Skolnick, R. Skladman, and A. S. Woo, "Comparative study of cranial anthropometric measurement by traditional calipers to computed tomography and threedimensional photogrammetry," J. Craniofac. Surg., vol. 24, no. 4, pp. 1106-1110, 2013, https://doi.org/10.1097/SCS.0b013e31828dcdcb.

[10]R. Muñoz-Salinas, M. J. Marín-Jimenez, E. Yeguas-Bolivar, and R. Medina-Carnicer, "Mapping and Localization from Planar Markers," Pattern Recognit., vol. 73, pp. 158-171, 2018, https://doi.org/10.1016/j.patcog.2017.08.010.

[11]M. Pierrot Deseilligny and I. Clery, "Apero, an Open Source Bundle Adjusment Software for Automatic Calibration and Orientation of Set of Images," ISPRS - Int. Arch. Photogramm. Remote Sens. Spat. Inf. Sci., vol. XXXVIII-5/W16, pp. 269-276, 2011, https://doi.org/10.5194/isprsarchives-XXXVIII-5-W16-269-2011.

[12]M. Kreutz et al., "Facial asymmetry correction with moulded helmet therapy in infants with deformational skull base plagiocephaly.," J. Craniomaxillofac. Surg., vol. 46, no. 1, pp. 28-34, 2018, https://doi.org/10.1016/j.jcms.2017.10.013.

[13] I. Barbero-García, J. L. Lerma, Á. Marqués-Mateu, and P. Miranda, "Low-Cost SmartphoneBased Photogrammetry for the Analysis of Cranial Deformation in Infants," World Neurosurg., vol. 102, pp. 545-554, 2017, https://doi.org/10.1016/j.wneu.2017.03.015. 